\title{
MEMS-Based Conductometric Sensors for Organic Airborne Particles
}

\author{
Naomichi Jimbo, Ren Horiuchi, Tomiharu Yamaguchi, Kazuhiro Hara \\ Tokyo Denki University, 5 Senjuasahi-cho, Adachi-ku, Tokyo 120-8551 Japan \\ hara@eee.dendai.ac.jp
}

\begin{abstract}
:
We have developed MEMS-based conductometric sensors for detection of smaller organic airborne particles such as PM2.5 and house dust which may cause a variety of diseases. A sensor is composed of two parts: a sensing element and a micro heater. Both parts were fabricated by using thin film technology, IC fabrication process and micromachining technique. The resistance of the sensor steeply decreased and then recovered to the initial value. A small increase appeared before or after the resistance decrease when a piece of PM2.5 and house dust adhered to the surface of the sensing film and burnt on it. The magnitude of the resistance decrease and the recovery time depended on the size of the organic airborne particles. In addition, the small increase of the resistance before or after the large decrease offered some information about the constituents of the particle. Thus it is possible to identify the species of the particle by the developed sensors.
\end{abstract}

Key words: organic airborne particles, PM2.5, house dust, MEMS-based sensor, metal oxide film

\section{Introduction}

In recent years, air pollution caused by various substances associated with industrial activities is becoming a serious problem. Particularly, airborne particles with very small sizes are easy to penetrate deep into the lungs, causing respiratory diseases such as asthma and bronchitis and increasing the possible risk of lung cancer[1].

On the one hand, in a house, allergic symptoms caused by house dust made up of carcasses and feces of mites floating in the air, mold spores and the like are becoming a big problem. Human nose can detect toxic gases and foul odors, but these organic airborne particles cannot be perceived. So, it is necessary to develop a sensor that detects organic airborne particles.

Several methods have been developed to monitor airborne materials. Various types of samplers along with filters are usually used to count the number of airborne particles. The gravitational method by a Durham's sampler is a common one to obtain the number of pollen. Particle counters with laser optics are sometimes used. An automatic measuring system based on beta ray absorption method is also used as a measuring instrument for regular monitoring. However, these methods need expensive and large equipment. Inexpensive conductometric sensors for detection of mold spores and pollen have been reported by one of the present authors[2][3].

This paper describes MEMS-based micro sensors that are capable of detecting smaller organic airborne particles such as PM2.5 and house dust.

\section{Sensor Configuration and Fabrication}

A developed sensor is shown in Fig. 1. The device is composed of two elements: a sensing element and a micro heater. A double-layered sensing film composed of $\mathrm{Fe}_{2} \mathrm{O}_{3}+\mathrm{TiO}_{2}$ (5 $\mathrm{mol} \%)+\mathrm{MgO}(4 \mathrm{~mol} \%)$ and $\mathrm{SnO}_{2}+\mathrm{V}_{2} \mathrm{O}_{5}(4$ mol\%) was used. Each of the two elements was fabricated on a substrate having a diaphragm structure. Au wires of $25 \mu \mathrm{m}$ in diameter were inserted between the two parts to make a gap which allow small particles to reach the sensing film.

The sensor was fabricated using thin film technology, IC fabrication process and MEMS technique. All the films were successively deposited by r.f. sputtering. A diaphragm structure was made by deep reactive-ion etching of Si from the backside. 


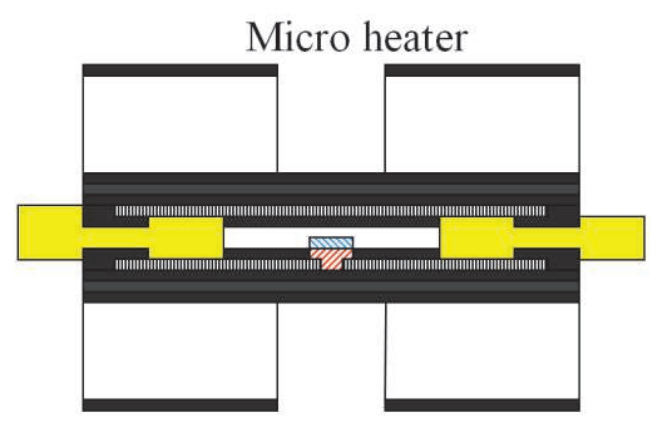

Sensing element

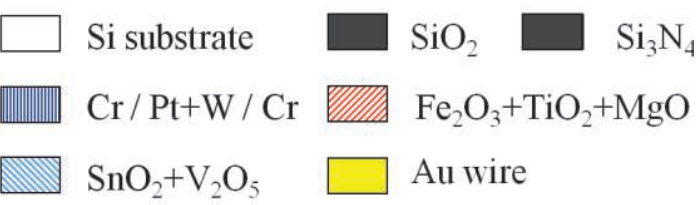

Fig. 1. Schematic cross-sectional view of a developed sensor.

\section{Sensing Principle}

The sensing principle for organic airborne particles is basically similar to that for reducing gases. When organic airborne particles adhere to the sensor surface and burn on it, they react with chemisorbed or lattice oxygen atoms on the surface of the metal oxide film, emitting electrons into the conduction band of the sensing film. As a result, the resistance value of the sensing film decreases. Then the resistance value gradually recovers to the initial one by adsorption of oxygen in the air after the particles burn out on the surface of the sensing film. Both the resistance decrease and recovery time are dependent on the particle size. Thus the particle size can be estimated from these measurements.

\section{Experimental Setup}

The sensor was tested in a closed box made of PMMA. The sensor resistance was measured using a digital multi-meter (Fluke, 287). The power consumption of the fabricated sensor ranged from $258 \mathrm{~mW}$ to $647 \mathrm{~mW}$. In this study, particles generated from a diesel engine and house dust were used as the object to be detected.

\section{Experimental Results and Discussion}

The sensor response to DPM (diesel particulate matter) and house dust are shown in Fig. 2 and 3 , respectively. The resistance change was observed depending on the particle size contained in the test samples. Moreover, small increase of the resistance before large decrease appeared several times in Fig. 2. This increase may result from combustion of sulfuric salt which is sometimes contained in DPM; the resultant $\mathrm{SO}_{2}$ molecule adsorbs as $\mathrm{SO}_{2}{ }^{-}$on the surface of the sensing film after capturing electron from it. Small increase of the resistance after large decrease appeared in Fig. 3 . This increase may result from amino acid contained in carcasses of mites; the resultant $\mathrm{NO}_{2}$ molecule similarly adsorbs as $\mathrm{NO}_{2}^{-}$on the surface of the sensing film after capturing electron from it. It takes more time to combust carcasses of mites because they are usually covered by insect cuticle. Thus the small increase of the resistance appears after large decrease.

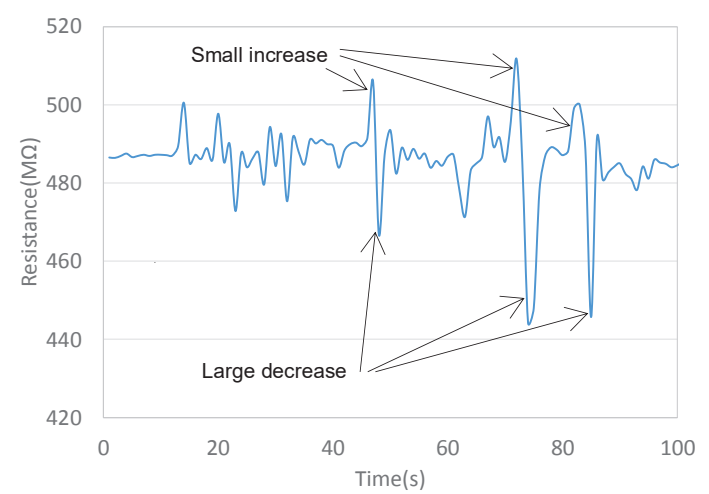

Fig. 2. Response to DPM.

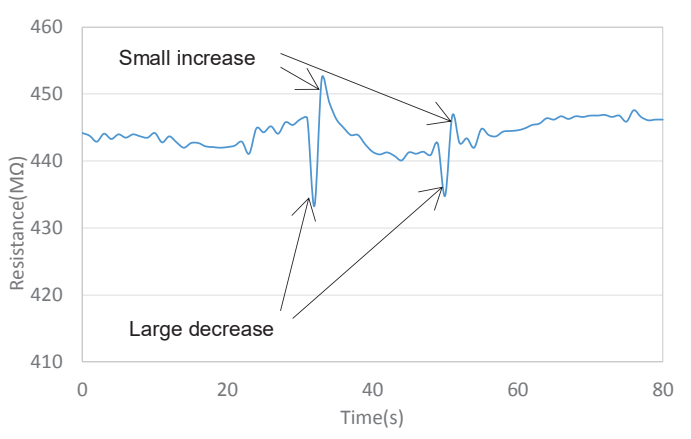

Fig. 3. Response to house dust.

\section{Acknowledgement}

The present work was supported by JSPS KAKENHI Grant Number JP16K06391.

\section{References}

[1] Shaolong Feng, Dan Gao, Fen Liao, Furong Zhou, Xinming Wang, The health effects of ambient $\mathrm{PM}_{2.5}$ and potential mechanisms, Ecotoxicology and Environmental Safety, 128, 67-74 (2016); doi: 10.1016/j.ecoenv.2016.01.030.

[2] K. Hara, Sensors for Monitoring Harmful Gases and Organic Floating Particles, Biochemical Sensors-Mimicking Gustatory and Olfactory Senses ed. by K. Toko, Pan Stanford Publishing Pte. Ltd., Singapore; ISBN 978-981-4267-07-6.

[3] K. Tsuruzoe, K. hara, Micro Sensors for Realtime Monitoring of Mold Spores and Pollen, Proc. of the Int. Conf. on Biomedical Electronics and Devices(BIODEVICES-2015), Lisbon, 174-179 (2015); doi: 10.5220/0005279301740179. 